\title{
Victimización en la escuela: relación de la crianza y variables funcionales-disfuncionales del desarrollo
}

\author{
Paula Samper-García ${ }^{*}$, Vicenta Mestre-Escrivá ${ }^{1}$, Elisabet Malonda ${ }^{1}$ y Belén Mesurado ${ }^{2}$ \\ 1 Departamento de Psicología Básica. Facultat de Psicologia. Universitat de València (España). \\ 2 Centro Interdisciplinario de Investigaciones en Psicología Matemática y Experimental (CIIPME). Buenos Aires (Argentina).
}

\begin{abstract}
Resumen: El presente estudio tiene como principal objetivo analizar el papel diferencial de la crianza (apoyo y comunicación versus control), de variables funcionales del desarrollo (empatía y mecanismos de afrontamiento funcionales) y las variables disfuncionales (inestabilidad emocional y afrontamiento improductivo) en la victimización ocurrida en el entorno escolar, o por el contrario, con el apego entre pares. Participaron en el estudio 418 alumnos, 224 chicas y 194 chicos adolescentes, entre 13 y 14 años, escolarizados en los niveles de $3^{\circ}$ y $4^{\circ}$ curso de Educación Secundaria Obligatoria. Para analizar estas relaciones se llevó a cabo un modelo de ecuaciones estructurales EQS. Los resultados obtenidos muestran que los estilos de crianza de apoyo y comunicación versus control negativo están directamente relacionados con las variables funcionales y disfuncionales de desarrollo. El control negativo aparece relacionado con la victimización. A su vez, las variables funcionales están directamente relacionadas tanto con la victimización como con el apego, mientras que las variables disfuncionales solo alcanzan una correlación significativa con la victimización. Finalmente se discuten los resultados y sus posibles implicaciones.

Palabras clave: Victimización; estilos de crianza; empatía; inestabilidad emocional; mecanismos de afrontamiento.
\end{abstract}

Title: Victimization at school: relationship of parenting and functionaldysfunctional developmental variables

Abstract: This study has the aim to analyze the different role of parental styles (affect and communication versus control), of functional developmental variables (empathy and adaptative coping styles) and dysfunctional variables (emotional instability and inefficient coping styles) in school victimization, or conversely with peer attachment. Participants were 418 Spanish adolescents, 224 girls and 194 boys, aged between 13 - 14 years old and attending year 7 and year 8 of secondary school. Structural equation modeling was carried out using the EQS program.

Results shows that parenting styles of affect and communication versus negative control are both directly related to functional and dysfunctional developmental variables. Negative control is also directly related to the school victimization. Also, functional variables are directly related to the school victimization and peer attachment, while dysfunctional variables only are related to school victimization. Finally, results and their possible implications are discussed.

Keywords: Victimization; peer attachment; parenting styles; empathy; emotional instability; coping styles.

\section{Introducción}

En la adolescencia las relaciones sociales con los compañeros adquieren una especial importancia por la relevancia que tienen para el adolescente y también por el impacto que estas relaciones tienen en el ajuste psicosocial de la persona. En el contexto escolar la aceptación o rechazo social por los iguales están relacionados con un mayor o menor equilibrio emocional y en general con la adaptación de los adolescentes.

La violencia perturba la convivencia y tiene importantes consecuencias negativas para la salud y el ajuste psicosocial de los menores (Cerezo, 2009; Garaigordobil y Oñaderra, 2010a). En concreto, el bullying es una forma de conducta agresiva, intencionada y perjudicial, cuyos protagonistas son jóvenes escolares; es además una conducta persistente. La mayoría de los agresores actúan motivados por un deseo de poder, de intimidar y dominar (Cerezo, 2006). En esta díada agresor - víctima, la víctima está indefensa, sin posibilidad de respuesta ni apoyos. Es, como algunos investigadores en el tema han señalado, un juego perverso de dominio-sumisión que cuando se mantiene de forma prolongada da lugar a procesos de victimización, con el consiguiente deterioro psicológico para la víctima (Garaigordobil, 2010).

La victimización implica, pues, la exposición repetida a una conducta hostil por parte de los compañeros. Se define como la experiencia de ser objeto de conductas de agresión

* Dirección para correspondencia [Correspondence address]:

Paula Samper García. Avda Blasco Ibáñez, 21. 46010. Valencia.

E-mail: paula.samper@uv.es física, verbal y psicológica, perpetradas por los iguales en el entorno escolar, especialmente en lugares con escasa supervisión por parte de los adultos (Graham, 2006; Olweus, 1993).

Por el contrario, la aceptación por parte de los compañeros es esencial para la socialización, el desarrollo de habilidades sociales, la interiorización de valores, la capacidad de autorregulación emocional; además, supone una fuente de apoyo emocional, de seguridad y de compañía (Escobar, Fernández-Baena, Miranda, Trianes y Cowie, 2011). Los adolescentes que se sienten aceptados por sus iguales amplían sus relaciones interpersonales y disponen de más recursos de apoyo, que a su vez contribuyen a un mayor bienestar y ajuste psicosocial (Estévez, Martínez y Jiménez, 2009).

En general, un clima escolar en el que el alumno se siente valorado y aceptado, caracterizado por el apoyo, la confianza y el respeto mutuo, configura un espacio de interacción social positivo. Por el contrario, los adolescentes victimizados perciben ese entorno escolar cargado de inseguridad y de desconfianza, lo que correlaciona con un pobre ajuste escolar (Martínez-Ferrer, Povedano-Díaz, Amador-Muñoz y Moreno-Ruiz, 2012).

\section{El rol de los padres en la victimización y el apego entre iguales}

Las características del entorno pueden ser factores de riesgo o de protección frente a la victimización, siendo el ambiente familiar en el que se desarrolla el menor especialmente importante, sobre todo por la gran influencia que 
ejerce en el desarrollo social y emocional de los niños y adolescentes (Piñero-Ruiz, López-Espín, Cerezo y TorresCantero, 2012).

Las pautas educativas de los padres y la calidad de las relaciones en el ámbito familiar constituyen factores relacionados con el ajuste psicosocial en la infancia y la adolescencia. Existe un cierto acuerdo en que el ambiente familiar juega un importante papel en el desarrollo de los hijos, en su disposición prosocial, en la empatía (Carlo, Mestre, Samper, Tur y Armenta, 2010a; Mestre, Samper, Nácher, Tur y Cortés, 2006; Ortiz, Apodaca, Etxebarría, Fuentes y López, 2008), en la conducta agresiva y la inestabilidad emocional (Oliva, Parra y Arranz, 2008; Tur-Porcar, Mestre, Samper y Malonda, 2012; Wahl, \& Metzner, 2012). En general, el ajuste psicosocial de los hijos e hijas es mayor en familias caracterizadas por el afecto, la comunicación y las normas en las relaciones padres- hijos.

En relación con el rol que desempeñan los estilos de crianza en la victimización, hay resultados contradictorios. Algunos estudios han encontrado que el apoyo paterno y las relaciones familiares no están relacionadas con la victimización y que los padres de chicos y chicas victimizados no se diferencian de aquellos padres cuyos hijos son víctimas de sus compañeros (Baldry, 2004; Beran, \& Violato, 2004). Sin embargo, otras investigaciones en población adolescente concluyen que la victimización y la conducta desviada están directamente relacionadas con estrés psicológico y que dicha relación está mediatizada por la comunicación entre los adolescentes y sus padres (Herrero, Estévez y Musitu, 2006).

Por otra parte, hay estudios que concluyen la relación entre el afecto parental, especialmente el apego establecido con la madre, con el apego a iguales a lo largo de la adolescencia, de manera que los chicos y chicas que formaron en la infancia un vínculo seguro en sus familias tienden a mantener este mismo tipo de apego en las relaciones con sus iguales (Delgado, Oliva y Sánchez-Queija, 2011).

\section{Variables cognitivas y emocionales}

Investigaciones recientes sobre victimización en el entorno escolar se han centrado en los procesos cognitivos y emocionales como reguladores y como mecanismos de control de la conducta agresiva y victimización entre pares (Anderson, \& Hunter, 2012). Las estrategias para afrontar y solucionar los problemas, la empatía, las habilidades sociales, la inteligencia emocional o el autocontrol en la interacción con los pares se consideran puntos fuertes o deficiencias para una buena adaptación social y unas relaciones sociales adecuadas (apego) con los compañeros (Petrides, Frederickson, \& Furnham, 2004).

Investigaciones anteriores concluyen una relación negativa entre bullying y empatía, especialmente en su componente cognitivo. La comprensión de las emociones de otros parece inhibir la expresión de conducta antisocial (Gini, Albiero, Benelli, \& Altoe, 2007).
También la empatía puede desempeñar un importante rol en las experiencias de victimización, porque una orientación empática hacia otros facilita la calidad de las relaciones interpersonales y puede promover el ajuste social (Malti, Perren, \& Buchmann, 2010).

Otros estudios han concluido que la victimización está relacionada con falta de regulación emocional y control de impulsos y con niveles bajos de empatía en su componente cognitivo (Kokkinos, \& Kipritsi, 2012). Por tanto, si la víctima es incapaz de reconocer, comprender y regular sus emociones, esto puede producir rechazo por parte de sus compañeros. Por el contrario, en dichos estudios, la victimización correlacionaba positivamente con el componente emocional de la empatía, por ejemplo, el compartir el estado emocional de otros. Estos resultados se interpretan como que la posición de ser sistemáticamente victimizado hace a la víctima más consciente de las disposiciones emocionales de sus agresores. Otros autores han señalado que el niño victimizado es más empático porque es particularmente sensible a las consecuencias emocionales de las transgresiones a las normas (Mavroveli, Petrides, Sangareau, \& Furnham, 2009). En general, la incapacidad de reconocer, comprender, manejar y expresar sus emociones o las de sus compañeros puede ser un fuerte predictor de ser una posible víctima (Kokkinos, \& Kipritsi, 2012).

En general, la empatía se considera una emoción controlada, orientada a los sentimientos del "otro" y por tanto, contrapuesta a la impulsividad o falta de autocontrol (Carlo, Mestre, Samper, Tur, \& Armenta, 2010b; Carlo, Mestre, McGinley, Samper, Tur, \& Sandman, 2012).

Además, la impulsividad se relaciona con otros procesos cognitivos como el afrontamiento y la capacidad para solucionar problemas (Arán-Filippetti y Richaud, 2011), de manera que una mayor capacidad de autocontrol ante los problemas facilita la planificación de una acción eficaz y la puesta en práctica de mecanismos de afrontamiento funcionales orientados a la solución del problema. En población infantil existen resultados que relacionan las conductas disruptivas con mecanismos de afrontamiento inadecuados que impiden conductas alternativas a la conducta inadaptada (IsonZintilini y Morelato, 2008). Los mecanismos de afrontamiento son procesos que influyen en las respuestas más o menos adaptadas a la situación que la persona vive en un momento dado. Estas estrategias de afrontamiento son especialmente importantes en la adolescencia, etapa en que la presión del grupo y la necesidad de autoafirmación hacen imprescindibles los recursos para afrontar un problema de manera adaptada y encontrar soluciones no violentas a los conflictos. Un estudio reciente en adolescentes españoles entre 12 y 15 años constata que los sujetos menos agresivos utilizan en situaciones conflictivas o que producen tensión estrategias propias de un estilo de afrontamiento centrado en la resolución del problema en la relación con los demás, mientras que el afrontamiento improductivo (autoinculparse, ignorar el problema, hacerse ilusiones...) es más frecuente en los adolescentes que puntúan más alto en agresividad. Se confirma así 
la relación entre afrontamiento y agresividad y el carácter protector que los estilos de afrontamiento centrados en la resolución del problema y en la relación con los demás ejercen frente a la agresividad (Mestre, Samper, Tur-Porcar, Richaud y Mesurado, 2012).

En el presente estudio hemos querido analizar el papel diferencial de la crianza y de variables cognitivas y emocionales sobre la victimización en el contexto escolar o sobre el apego entre los pares en la temprana adolescencia. Se trata de establecer en qué medida el apoyo y la comunicación versus el control estricto ejercido por el padre y la madre en las relaciones con sus hijos, junto las variables funcionales del desarrollo (empatía y mecanismos de afrontamiento funcionales) y las variables disfuncionales (inestabilidad emocional y afrontamiento improductivo) guardan relación con la victimización ocurrida en el entorno escolar, o por el contrario, con el apego entre pares. El objetivo central, es pues, analizar la interacción entre los dos estilos de crianza mencionados y determinados procesos cognitivos y emocionales, para analizar su relación con la victimización o el apego con los compañeros. Se trata de poder concluir los procesos más relacionados con la victimización de los adolescentes y con el apego entre iguales, que apoyen propuestas orientadas a la prevención e intervención en este ámbito. Si bien hay investigaciones que analizan la importancia de los estilos de crianza en el ajuste psicosocial de los adolescentes y otras más centradas en procesos cognitivos y emocionales, disponemos de menos estudios que analicen el rol de los padres junto con variables que influyen directamente en las relaciones sociales y por tanto pueden afectar la victimización en el entorno escolar o por el contrario favorecer el apego entre pares.

Tomando como base las investigaciones previas planteamos las siguientes hipótesis:

1) Los adolescentes que perciban un alto apoyo y comunicación por parte de sus padres (padre y madre) desarrollarán un mayor apego con sus compañeros.

2) Los adolescentes que perciban un control negativo y estricto en las relaciones con sus padres (padre y madre) alcanzarán mayores niveles de victimización en el entorno escolar.

3) Los adolescentes que perciban más apoyo y comunicación por parte de sus padres serán más empáticos y tendrán mecanismos de afrontamiento más funcionales y adaptativos (variables funcionales del desarrollo). Esto a su vez favorecerá el apego con sus compañeros y les protegerá de la victimización.

4) Los adolescentes que perciban más control negativo por parte de sus padres serán más inestables emocionalmente y tendrán mecanismos de afrontamiento improductivo (variables disfuncionales del desarrollo). Esto a su vez favorecerá la victimización e inhibirá el apego con los compañeros.

\section{Método}

\section{Participantes}

La muestra está formada por 418 alumnos, 224 mujeres y 194 varones, entre 13 y $14(M=13.93 ; D T=.267)$ años, escolarizados en los niveles de $3^{\circ}$ y $4^{\circ}$ curso de Educación Secundaria Obligatoria. La muestra ha sido seleccionada aleatoriamente entre la población escolarizada en dichos niveles educativos en Centros Públicos ( 7 centros) y Concertados (4 centros) de la Comunidad Valenciana. En total han participado 11 Centros escolares.

\section{Instrumentos}

Para evaluar las variables objeto de estudio se utilizaron los siguientes instrumentos con adecuadas garantías psicométricas de fiabilidad y validez.

Cuestionario de estilos parentales adaptado del Child Reports of Parental Behavior Inventory (Schaefer, 1965; Samper, Cortés, Mestre, Nácher y Tur, 2006). Consta de 38 ítems dirigidos a evaluar los estilos de crianza que marcan las relaciones paterno-filiales según la percepción de los hijos. Ejemplos de ítems son: "Le gusta hablar conmigo", "A menudo me alaba" o "Pierde el control conmigo cuando no sigo su consejo". Los participantes indicaron su acuerdo con algunas situaciones familiares en una escala $(1=$ total acuerdo, $2=$ algunas veces, 3 = totalmente diferente). El menor ha de contestar pensando en la conducta del padre y de la madre de forma diferenciada. Los factores que evalúa son: Apoyo y comunicación, expresa el sentimiento de apoyo emocional por parte de los padres, se envían mensajes a los hijos e hijas de afecto y apoyo, fomentando la autonomía sobre la base de criterios disciplinarios y con un alto nivel de comunicación entre padres e hijos. Control negativo, describe las relaciones basadas en el control estricto, la irritabilidad y la evaluación negativa y de rechazo hacia el hijo. Permisividad y Negligencia, basadas en la autonomía extrema, con ausencia de normas y de criterios disciplinarios donde todo está permitido, lo que lleva a los hijos a percibir una falta de atención hacia sus necesidades. Las escalas muestran adecuados índices de fiabilidad (Apoyo y comunicación madre: alfa $=.88$ y padre alfa $=, 89 ;$ Control negativo madre alfa $=.80$ y padre alfa $=.78 ;$ Negligencia madre alfa $=$ .56 y padre alfa $=.62$ y Permisividad madre alfa $=.41$ y padre alfa $=.47)$. En el presente estudio hemos utilizado únicamente el factor de Apoyo y comunicación y el factor de Control negativo.

Escala de Inestabilidad Emocional (Caprara, \& Pastorelli, 1993; Del Barrio, Moreno y López, 2001). Describe la conducta que indica una falta de autocontrol en situaciones sociales como resultado de la escasa capacidad para frenar la impulsividad y emocionalidad. Ejemplo ítems: "Interrumpo a los demás cuando hablan", "No puedo estar quieto/a". La escala alcanza una fiabilidad adecuada (alfa $=.80)$.

The Interpersonal Reactivity Index (IRI) (Davis, 1980; Mestre, Frías y Samper, 2004). Este instrumento evalúa la disposi- 
ción empática a través de dos factores emocionales (Preocupación empática y Malestar Personal) y dos cognitivos (Toma de perspectiva y Fantasía). Cada factor tiene 7 ítems como: "Me preocupan los problemas de los demás" (preocupación empática; alfa $=.60)$, "Cuando debo decidirme, escucho diferentes opiniones" (toma de perspectiva; alfa $=.62$ ) "Me identifico con los personajes de una novela" (Fantasía; alfa $=.64$ ) and "Cuando veo a alguien herido, me pongo nervioso" (malestar personal; alfa $=.56$ ) en una escala de 5 puntos desde 1 (no me describe bien) a 5 (me describe muy bien). Diversos estudios han mostrado propiedades psicométricas similares del IRI con muestras de estudiantes americanos, europeos y españoles (Davis, 1983; Eisenberg, Carlo, Murphy, \& Van Court, 1995; Mestre, Samper y Frías, 2002). Para el presente estudio hemos utilizado los dos factores más importantes del instrumento: toma de perspectiva y preocupación empática.

Escala de Afrontamiento para Adolescentes (Frydenberg, \& Lewis, 1995; Pereña y Seisdedos, 1997). Evalúa cómo el adolescente de 12 a 18 años afronta sus problemas en general. Concretamente, se diferencian 18 estrategias de afrontamiento: Buscar apoyo social; Concentrarse en resolver el problema; Esforzarse y tener éxito; Preocuparse; Invertir en amigos íntimos; Buscar pertenencia; Hacerse ilusiones; La estrategia de falta de afrontamiento; Reducción de la tensión; Acción social; Ignorar el problema; Autoinculparse; Reservarlo para sí; Buscar apoyo espiritual; Fijarse en lo positivo; Buscar ayuda profesional; Buscar diversiones relajantes y Distracción física. Estas estrategias pueden agruparse en tres estilos básicos de afrontamiento: Dirigido a la resolución del problema (alfa $=.76$ ), afrontamiento en relación con los demás (solicitud de ayuda) (alfa $=.85$ ) y afrontamiento improductivo (alfa $=.81)$.

Kit at School (KS, Buhs, McGinley, \& Toland, 2010). En esta investigación se han utilizado 6 de los ítems que recogen los tres factores sobre victimización descritos en la escala de Buhs, McGinley, \& Toland (2010) que hacen referencia a victimización relacional (hace referencia a las conductas que pretenden lesionar a través de "manipulación intencional y daño de la relación entre iguales"), manifiesta (incluye comportamientos físicos (por ejemplo, golpes) y verbales (por ejemplo, insultos) destinados a perjudicar directamente a los demás; y exclusión social. Los estudiantes han de contestar en una escala Likert de 5 alternativas de respuesta $(1=$ "casi nunca”, 3 =algunas veces y $5=$ "casi siempre”). Ejemplos de ítem son ¿Con qué frecuencia los/las compañeros/as en tu centro: "se burlan de ti o te insultan?" (manifiesta), “¿cuentan mentiras, chismes, rumores o difunden malas noticias sobre ti?" (relacional), "¿te dejan fuera de las conversaciones, juegos o actividades? (exclusión social)". El índice de fiabilidad alfa de Cronbach es de .70 .

Acercamiento a Pares (tomado del IPPA, Inventory of Parent and Peer Attachment, de Armsden, \& Greenberg, 1987). Evalúa las dimensiones conductuales y afectivo/cognitivas de apego de los adolescentes a sus compañeros. Ejemplo ítems: "Mis amigos/as respetan mis sentimien- tos", "Les cuento a mis amigos/as mis problemas y dificultades", "Si mis amigos/as saben que algo me preocupa, me preguntan por ello". El índice de fiabilidad alfa de Cronbach es de .75 .

\section{Procedimiento}

Una vez realizada la selección aleatoria de los centros escolares, se solicitó autorización a la Dirección General de Centros de la Consellería de Educación de la Comunidad Valenciana para acceder a los mismos. El contacto con los equipos directivos se realizó primero a través de un escrito a cada uno de los Directores en el que se les informaba del contenido de la investigación, de los objetivos y de la población objeto de estudio, así mismo, se solicitaba su colaboración. A continuación, se concertaron visitas a los centros para poder informar directamente al equipo directivo y al profesorado implicado en los niveles educativos a evaluar. Las entrevistas sirvieron a su vez para solucionar posibles dudas y planificar el cronograma de evaluación. Una vez aceptada la colaboración por parte de la dirección del centro, se informó de la investigación a los padres a través de una carta, en la que además se solicitaba la autorización paterna para la evaluación de su hijo o hija.

Obtenidos todos los permisos, en cada centro educativo se aplicaron los cuestionarios de forma colectiva y en horas lectivas en cada aula y para cada uno de los niveles de edad y cursos seleccionados. El pase de las pruebas se llevó a cabo en una sesión de una hora aproximadamente. Los evaluadores entrenados para esta investigación explicaron en cada aula los instrumentos de evaluación, las instrucciones para cumplimentarlos y resolvieron las dudas de los alumnos, insistiendo en el anonimato de los cuestionarios y la importancia de la sinceridad en las respuestas.

Para la realización del estudio se siguieron todas las normas éticas, respetando el anonimato de las respuestas, tanto para la recogida de datos como en el tratamiento posterior de los mismos.

\section{Análisis de datos}

En primer lugar, se han realizado los análisis descriptivos y correlacionales de las variables objeto de estudio. A continuación, se realizaron dos Modelos de Ecuaciones Estructurales (SEM) para explorar los Modelos de Victimización y de Relaciones con los Pares usando el programa estadístico AMOS 16.0 (SPSS Inc. 2007). Se usaron los siguientes índices de bondad de ajuste: el Chi cuadrado, la razón del Chi cuadrado en los grados de libertad $\left(\chi^{2} / g l\right)$, el índice de bondad de ajuste (goodness of fitindex - GFI), el índice de bondad de ajuste ajustado (adjusted goodness of fitindex AGFI), y finalmente la Raíz media residual (Root mean residual - RMR) que es un índice de medición del error. 


\section{Resultados}

\section{Análisis descriptivos}

La Tabla 1 muestra la estadística descriptiva y las correlaciones de las variables incluidas en el estudio: Control del Padre y de la Madre, Apoyo y Comunicación del Padre y de la Madre, Variables del desarrollo Funcionales (Empatía Toma de perspectiva, Preocupación Empática- y afrontamiento funcional -Resolución de Problema y Solicitud de
Ayuda-), Variables del desarrollo Disfuncionales (Inestabilidad Emocional y Afrontamiento Improductivo), Victimización y Relación de Apego con los Pares.

Las correlaciones, tal como figuran en la Tabla 1 , indican una relación positiva y significativa entre cada una de las dimensiones de los estilos de crianza en lo referente al padre y a la madre, así como una relación también significativa, pero inversa entre la dimensión de apoyo y comunicación y la dimensión de control.

Tabla 1. Correlaciones de las variables incluidas en el estudio.

\begin{tabular}{|c|c|c|c|c|c|c|c|c|c|c|c|c|}
\hline & 1 & 2 & 3 & 4 & 5 & 6 & 7 & 8 & 9 & 10 & 11 & 12 \\
\hline 1. Apoyo y comunicación Madre & - & & & & & & & & & & & \\
\hline 2. Apoyo y comunicación Padre & $.61 * *$ & - & & & & & & & & & & \\
\hline 3. Control Madre & $-.22 * *$ & $-.19 * *$ & - & & & & & & & & & \\
\hline 4. Control Padre & -.10 & $-.23 * *$ & $.65^{* *}$ & - & & & & & & & & \\
\hline 5. Toma de perspectiva & $.21 * *$ & $.18^{* *}$ & .08 & -.03 & - & & & & & & & \\
\hline 6. Preocupación empática & $.14^{* *}$ & $.14^{* *}$ & .03 & -.03 & $.49 * *$ & - & & & & & & \\
\hline 7. Resolución de problemas & $.30 * *$ & $.18^{* *}$ & -.01 & -.02 & $.26^{*}$ & $.15^{* *}$ & - & & & & & \\
\hline 8. Solicitud de ayuda & $.18^{* *}$ & $.11^{*}$ & .04 & .04 & $.22 * *$ & $.18^{* *}$ & $.54 * *$ & - & & & & \\
\hline 9. Afrontamiento improductivo & -.01 & -.09 & $.20^{* *}$ & $.19 * *$ & .06 & $.11^{*}$ & $.12^{*}$ & $.30^{* *}$ & - & & & \\
\hline 10. Inestabilidad emocional & $-.17 * *$ & $-.18^{* *}$ & $.21 * *$ & $.18^{* *}$ & $-.17 * *$ & -.10 & -.10 & .04 & $.22 * *$ & - & & \\
\hline 11. Victimización & $-.14 * *$ & $-.19 * *$ & $.26 * *$ & $.29 * *$ & -.02 & $-.16^{* *}$ & $-.16^{* *}$ & -.05 & $.23 * *$ & $.10^{*}$ & - & \\
\hline 12. Apego Pares & $.25^{* *}$ & $.20 * *$ & $.11 *$ & .06 & $.32 * *$ & $.20 * *$ & $.20 * *$ & $.33^{* *}$ & -.03 & -.07 & $-.32 * *$ & - \\
\hline$M$ & 2.19 & 2.10 & 1.83 & 1.77 & 3.27 & 3.56 & 3.29 & 2.74 & 2.47 & 1.69 & 1.51 & 3.75 \\
\hline DT & .37 & .39 & .41 & .37 & .66 & .62 & .46 & .46 & .41 & .33 & .54 & .52 \\
\hline
\end{tabular}

Nota: $* p<.01 ; * * p<.001$

Por otra parte, la empatía, tanto en su factor cognitivo (toma de perspectiva) como en su factor emocional (preocupación empática) alcanzan una correlación positiva con la relación de apoyo y comunicación, tanto por parte del padre como de la madre. Este resultado coincide con otros estudios que concluyen el importante papel que desempeñan los estilos de crianza en el desarrollo de la empatía y la disposición prosocial en los hijos (Carlo et al., 2010a; Mestre et al., 2006; Mestre, Tur, Samper, Nácher y Cortés, 2007; Ortiz et al., 2008).

Los mecanismos de afrontamiento funcional, centrados en la resolución del problema y en la solicitud de ayuda muestran una correlación positiva con las dos dimensiones de la empatía, y con el apoyo y comunicación percibido por los adolescentes en la relación con su padre y con su madre. Sin embargo, la relación no es significativa con la dimensión de control. Por el contrario, es el afrontamiento improductivo el que alcanza una correlación positiva y significativa con dicha dimensión de control ejercida por el padre y por la madre.

En la misma línea, la inestabilidad emocional también correlaciona altamente con dicho mecanismo de afrontamiento improductivo, así como con el control ejercido por el padre y la madre, mientras que alcanza una correlación negativa con el apoyo y la comunicación en la relación con los progenitores y con la dimensión cognitiva de la empatía.

Finalmente, la victimización correlaciona significativamente con los estilos de crianza, en negativo con el apoyo y la comunicación por parte de los padres, y en positivo con el control negativo. Además, alcanza una correlación negativa con la preocupación empática y con el mecanismo de afrontamiento centrado en la resolución del problema, mientras que correlaciona positivamente con el afrontamiento improductivo y con la inestabilidad emocional. Se constata, pues, la relación significativa entre la victimización, los estilos de crianza, la empatía, la inestabilidad emocional y los mecanismos de afrontamiento.

En sentido contrario figuran las correlaciones entre el apego con los pares y las variables evaluadas. La correlación es positiva con el apoyo y la comunicación con el padre y la madre y con las dos dimensiones de la empatía (toma de perspectiva y preocupación empática), así como, con los mecanismos de afrontamiento funcionales (resolución de problemas y solicitud de ayuda); mientras que la correlación es negativa con la victimización. Estos resultados constatan también una relación entre el apego con los pares y las variables evaluadas y la relación inversa entre la victimización y el apego.

\section{Modelo de ecuaciones estructurales - Modelo Victimización}

El primer modelo estudia la influencia de los estilos parentales tanto positivos (Amor y Comunicación materna y paterna) como negativos (Control materno y paterno) sobre variables funcionales del desarrollo referentes a la empatía (Toma de perspectiva, Preocupación Empática), a los meca- 
nismos de afrontamiento (Resolución de Problema, Solicitud de Ayuda) y a variables disfuncionales del desarrollo (Inestabilidad Emocional y Afrontamiento Improductivo) así como, la influencia de ambos sobre la percepción que los adolescentes tienen de ser victimizados. En la Figura 1 se presenta el modelo de ecuaciones estructurales con sus respectivos coeficientes de path. El modelo mostró un buen ajuste entre el modelo propuesto y los datos empíricos. Se obtuvo un $\chi^{2}$ $(36)=216.36 ; p<.000 ; \chi^{2} / g l=6$; GFI = .92; AGFI = .85; $\mathrm{CFI}=.82$ y RMR $=.02$, como se sabe valores por debajo de .10 indican un error aceptable y valores alrededor de .06 indican un muy buen ajuste (Hu, \& Bentler, 1999).

El modelo mostró que el estilo de crianza parental caracterizado por el apoyo y la comunicación favorece diferentes variables funcionales del desarrollo durante la adolescencia, tales como la empatía (tanto a nivel cognitivo -toma de perspectiva-, como emocional -preocupación empática-) y el afrontamiento funcional (estrategias orientadas a la resolución de problema y a la solicitud de ayuda), sin embargo no tiene una influencia sobre las variables disfuncionales del desarrollo incluidas en el estudio. Por otro lado, el estilo de crianza parental caracterizado por el exceso de control con ausencia de apoyo y comunicación tiene una importante influencia en el desarrollo de variables disfuncionales del desarrollo tales como el afrontamiento improductivo y la inestabilidad emocional, pero no mostró tener influencia sobre las variables funcionales. Por último, el modelo muestra que el control parental y las variables disfuncionales del desarrollo favorecen la percepción del adolescente de ser victimizado por sus compañeros, mientras las variables funcionales del desarrollo -tales como la empatía y el afrontamiento funcional- contribuyen a contrarrestar la percepción de los adolescentes de ser victimizados.

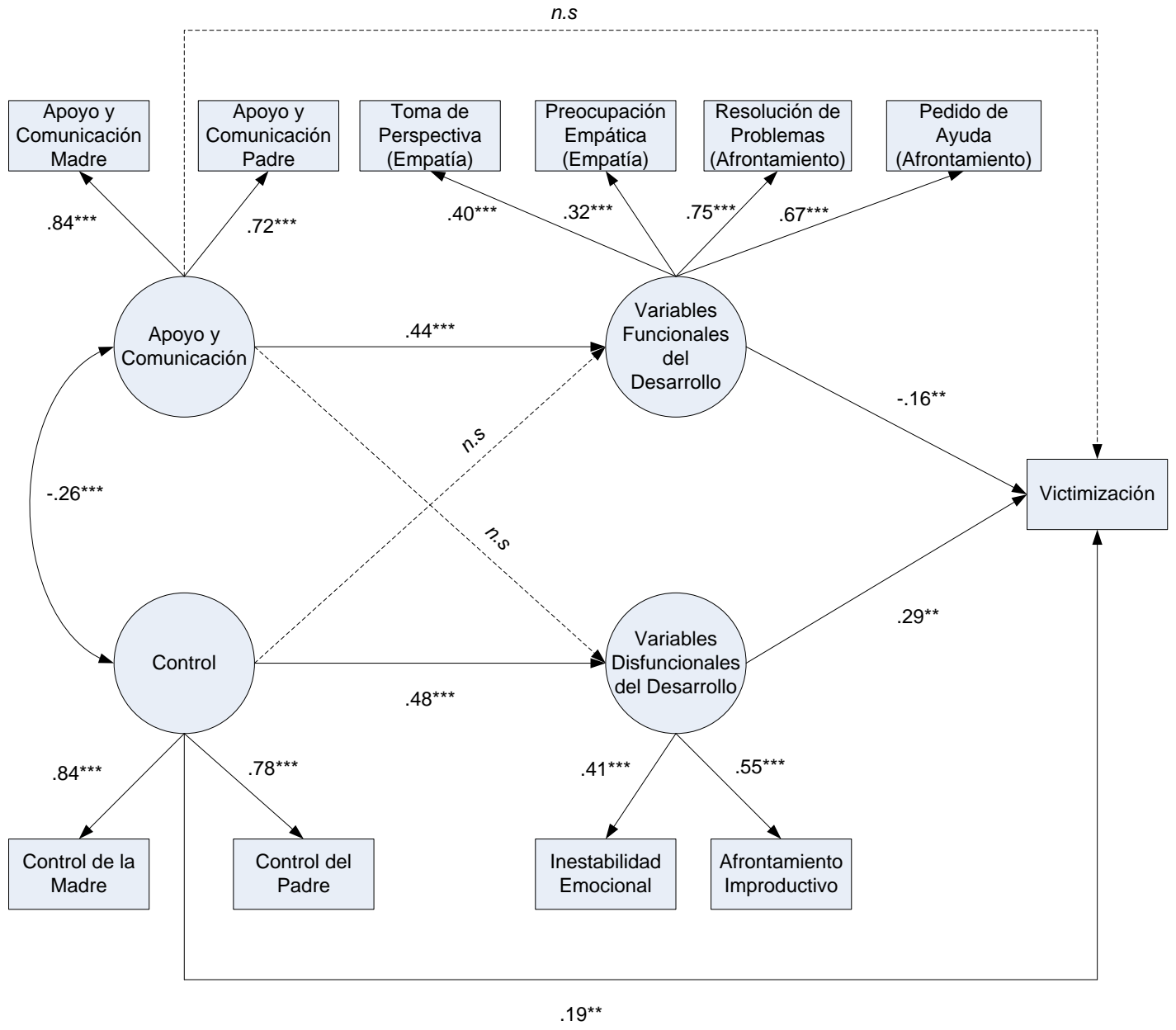

Figura 1. Modelo Victimización.

\section{Modelo de ecuaciones estructurales - Modelo Apego a los Pares}

El segundo modelo estudia la influencia de los estilos parentales tanto positivos (Amor y Comunicación materna y paterna) como negativos (Control estricto materno y paterno) sobre variables funcionales (Toma de perspectiva, Preocupación Empática, Resolución de Problema, Pedido de Ayuda) y disfuncionales del desarrollo (Inestabilidad Emo- 
cional y Afrontamiento Improductivo) así como, la influencia de estas variables sobre la percepción de apoyo de los pares. En la Figura 2 se presenta el modelo de ecuaciones estructurales con sus respectivos coeficientes de path. El modelo mostró un buen ajuste entre el modelo propuesto y los datos empíricos. Se obtuvo un $\chi^{2}(36)=234.5 ; p<.000 ; \chi^{2} / g l=$ 6 ; $\mathrm{GFI}=.91 ; \mathrm{AGFI}=.83$; $\mathrm{CFI}=.80$ y RMR $=.02$

$\mathrm{Al}$ igual que el anterior, el modelo mostró que el estilo de crianza parental caracterizado por el apoyo y la comunicación favorecen diferentes variables funcionales del desarrollo durante la adolescencia tales como la empatía (toma de perspectiva y preocupación empática) y el afrontamiento (resolución de problema y pedido de ayuda), sin embargo no tiene una influencia sobre las variables disfuncionales del desarrollo incluidas en el estudio. Por otro lado, el estilo de crianza parental caracterizado por el exceso de control con ausencia de apoyo y afecto tiene una importante influencia en el desarrollo de variables disfuncionales del desarrollo tales como el afrontamiento improductivo y la inestabilidad emocional pero no mostró tener influencia sobre las variables funcionales del desarrollo. El modelo mostró que el apego con los pares está solamente asociado a las variables funcionales del desarrollo (empatía y afrontamiento funcional) pero no a las variables disfuncionales del desarrollo, ni se encuentra asociado directamente a los estilos parentales de crianza.

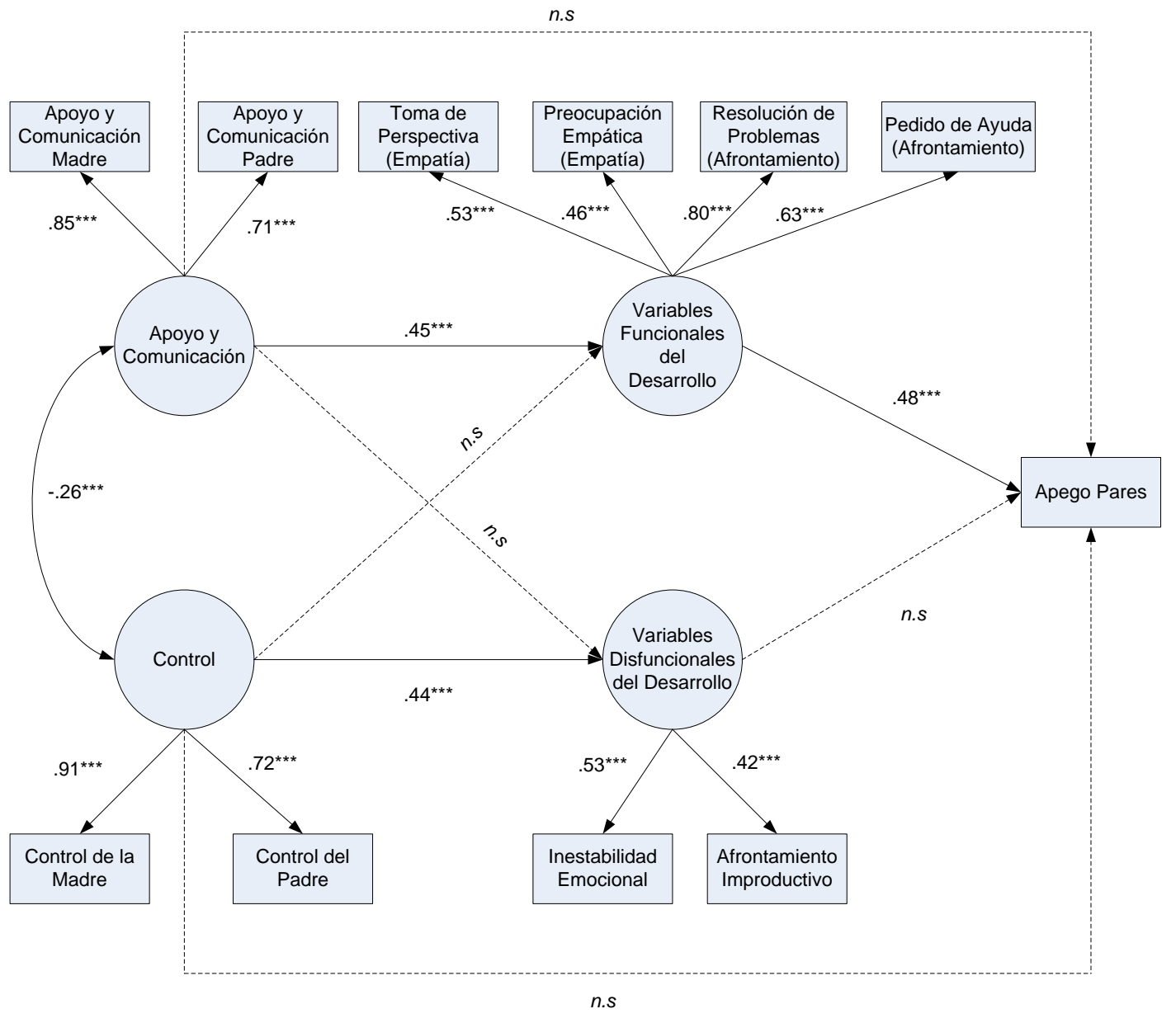

Figura 2. Modelo Apego Pares.

\section{Discusión}

La literatura científica sobre los estilos de crianza y el desarrollo psicosocial en la adolescencia, ha demostrado una asociación directa del afecto y la comunicación entre padres e hijos y un mejor desarrollo emocional y social en los adolescentes, mientras que un control excesivo está más relacionado con la agresividad y la desajuste social. El presente estu- dio ayuda a clarificar esta relación en concreto con la victimización en el entorno escolar y con el apego entre iguales, a través de la inclusión de variables funcionales del desarrollo, como la empatía y los mecanismos de afrontamiento adaptativos (orientados a la resolución del problema y a la solicitud de ayuda) o de variables disfuncionales, como la inestabilidad emocional y el afrontamiento improductivo. 
Los análisis correlacionales apoyan una relación entre el estilo de crianza del padre y la madre caracterizado por el apoyo y comunicación, así como la relación entre el control negativo ejercido por el padre y por la madre; por tanto, los adolescentes evaluados perciben esas dimensiones de la vida familiar de manera similar en sus progenitores. Además, la correlación negativa entre victimización y apego con los compañeros indica que son dos constructos contrapuestos y se trata de analizar qué variables influyen en uno y otro, y por tanto, pueden potenciar el apego e inhibir la victimización.

En relación con las hipótesis planteadas, las ecuaciones estructurales no aportan una relación directa de los estilos de crianza de apoyo y comunicación, ni de control negativo con el apego entre pares. Tan solo el control evaluado en la relación con el padre y con la madre alcanza una correlación significativa con la victimización. Estos resultados apoyan la segunda hipótesis y rechazan la primera hipótesis, ya que el afecto y la comunicación no guarda una relación directa con el apego con los compañeros. Otros estudios han indicado que el apoyo paterno no está relacionado con la victimización y que los padres de adolescentes victimizados no se diferencian de los padres cuyos hijos son víctimas de sus compañeros (Baldry, 2004; Beran, \& Violato, 2004). Nuestros resultados sí que apoyan que un mayor control negativo por parte de los padres favorece la victimización, mientras que no se constata esta relación en el caso del afecto y la comunicación.

El afecto del padre y de la madre correlacionan significativamente con el apego a los compañeros, pero a través de las variables funcionales del desarrollo (Delgado et al., 2011). De manera que el estilo de crianza caracterizado por el apoyo y comunicación en la relación con ambos progenitores, que transmite a los hijos mensajes de afecto y de valoración positiva, favorece el desarrollo de la empatía, tanto como capacidad para ponerse en el lugar del otro, como preocupación por el otro; así mismo, favorece también mecanismos de afrontamiento adaptativos ante un conflicto o problema, como son los orientados a la resolución del problema o los orientados a la búsqueda de ayuda. Todas estas variables funcionales influyen positivamente en el apego a los pares, mientras que dichas variables funcionales inhiben la victimización. Estos resultados confirman la tercera hipótesis.

Los adolescentes que perciben en la relación con su padre y con su madre un control negativo, caracterizado por la evaluación negativa y rechazo del hijo, a su vez son más inestables emocionalmente, tienen más dificultad para controlar sus impulsos y regular sus emociones; así mismo, utilizan mecanismos de afrontamiento improductivos, poco eficaces a la hora de afrontar un conflicto y encontrar soluciones al mismo. Estas variables disfuncionales del desarrollo favorecen la victimización en el entorno escolar, pero no guardan relación con el apego a los pares. Estos datos confirman parcialmente la hipótesis 4 . Se constata que las variables funcionales del desarrollo están directamente relacionadas con la victimización (en sentido negativo) y con el apego (en senti- do positivo) y también median entre el estilo de crianza de apoyo y comunicación y ambos constructos. Por el contrario, las variables disfuncionales están directamente relacionadas con la victimización, pero no con el apego, además, dichas variables median también en la relación entre el control y la victimización.

Podemos concluir el importante papel que desempeña el control negativo ejercido por el padre y por la madre en el desarrollo de la inestabilidad emocional y del afrontamiento improductivo y, a través de estas variables disfuncionales, en la victimización; pero también la relación directa entre dicho control y la victimización percibida por los adolescentes.

En resumen, los estilos de crianza de apoyo y comunicación versus control están directamente relacionados con las variables funcionales y disfuncionales del desarrollo evaluadas, pero no guardan una relación directa con el apego a pares, y tan solo el control negativo aparece directamente relacionado con la victimización. A su vez, las variables funcionales están relacionadas tanto con la victimización como con el apego, mientras que las variables disfuncionales solo alcanzan una correlación significativa con la victimización.

Se confirma, por tanto, la necesidad de incluir la empatía, la regulación emocional y estrategias de afrontamiento entre los factores relacionados con el ajuste psicológico y social y en concreto en la prevención de la victimización en el entorno escolar y en el desarrollo de relaciones de apego con los compañeros (Kokkinos, \& Kipritsi, 2012; Sánchez, Ortega y Menesini, 2012). La capacidad de reconocer y manejar las emociones que sentimos, de reconocerlas en los demás y de conectarnos afectivamente con ellos modula la calidad de las relaciones interpersonales que establecemos, de ahí la importancia de la empatía y la regulación emocional en el ajuste psicosocial. En el caso de las víctimas se constatan dificultades en la regulación emocional, en el uso de estrategias de afrontamiento más o menos eficaces para afrontar el problema, así como una baja empatía (Escobar et al., 2011; Kokkinos, \& Kipritsi, 2012).

Estas conclusiones tienen importantes repercusiones para la intervención directa en la adolescencia.

El control excesivo por parte de los progenitores puede favorecer el desarrollo de estrategias de afrontamiento ineficaces para afrontar los conflictos y una mayor impulsividad o dificultad de autocontrol, que constituyen habilidades adecuadas y necesarias ante situaciones de victimización. Por tanto, las pautas educativas de los padres y la calidad de las relaciones en el núcleo familiar deben ser tenidas en cuenta en los programas de prevención e intervención en violencia escolar, así como para favorecer relaciones de apego con los compañeros.

Además, la relación directa de las variables funcionales del desarrollo evaluadas con la victimización y el apego tienen implicaciones prácticas y sugieren la necesidad de implementar programas de intervención en contextos educativos que estimulen la empatía, la regulación emocional y estrategias de afrontamiento adaptativas, orientadas a la solución de los conflictos, ya que pueden contribuir al desarrollo 
de relaciones positivas y de apoyo entre los compañeros, así como a disminuir el número de niños y adolescentes que se sienten victimizados por sus iguales. Además, los programas de carácter preventivo tienen especial importancia en la Educación Primaria y primeros cursos de Educación Secundaria Obligatoria, dado el aumento de esta problemática a lo largo de estos niveles educativos (Garaigordobil y Oñaderra, 2010b; Sánchez et al., 2012).

Este estudio tiene varias limitaciones. En primer lugar, se trata de un estudio transversal y por tanto no permite establecer relaciones causales. Son necesarios estudios longitudinales futuros que permitan establecer las diferencias y evolución de las variables evaluadas. En segundo lugar, la evaluación se ha basado en autoinformes realizados por los adolescentes, en estudios futuros se puede contrastar la información de los padres con la de los adolescentes en relación con los estilos de crianza percibidos. No obstante, nuestro inte-

\section{Referencias}

Anderson, S., \& Hunter, S. C. (2012). Cognitive appraisals, emotional reactions, and their associations with three forms of peer-victimization. Psicothema, 24(4), 621-627. Recuperado de www.psicothema.com/pdf/4063.pdf.

Arán- Filippetti, V. y Richaud de Minzi, M. C. (2011). Efectos de un programa de intervención para aumentar la reflexividad y la planificación en un ámbito escolar de alto riesgo por pobreza. Universitas Psychologica, 10(2), 341-354. Recuperado de: http:/ /www.redalyc.org/articulo.oa?id $=64722451003$.

Armsden, G. C., \& Greenberg, M. T. (1987). The Inventory of Parent and Peer Attachment: Individual Differences and Their Relationship to Psychological Well-Being in Adolescence. Journal of Youth and Adolescence, 16(5), 427-454. doi: 10.1007/BF02202939.

Baldry, A. (2004). The impact of direct and indirect bullying on the mental and physical health of Italian youngsters. Aggressive Behavior, 30, 343355. doi: 10.1002/ab.20043.

Beran, T., \& Violato, C. (2004). A model of childhood perceived peer harassment: Analyses of the Canadian national longitudinal survey of children and youth Data. The Journal of Psychology, 138, 129-147. Recuperado en www.europepmc.org/abstract/.../15218785.

Buhs, E. S., McGinley, M., \& Toland, M. D. (2010). Overt and Relational Victimization in Latinos and European Americans: Measurement Equivalence Across Ethnicity, Gender, and Grade Level in Early Adolescent Groups. Journal of Early Adolescence, 30(1), 171-197. doi: $10.1177 / 0272431609350923$.

Caprara, G. V., \& Pastorelli, C. (1993). Early emotional instability, prosocial behaviour, and aggression: Some methodological aspects. European Journal of Personality, 7(1), 19-36. doi: 10.1002/per.2410070103.

Carlo, G., Mestre, V., McGinley, M., Samper, P., Tur, A., \& Sandman, D. (2012).The interplay of emotional instability, empathy, and coping on prosocial and aggressive behaviors. Personality and Individual Differences, 53, 675-680 doi: 10.1016/j.paid.2012.05.022.

Carlo, G., Mestre, M. V., Samper, P., Tur, A. M., \& Armenta, B. (2010a). Empathy and prosocial moral reasoning as mediators of the longitudinal relations between parenting styles and prosocial behaviours. Intenational Journal of Behavioral Development, 35(2), 116-124. doi: $10.1177 / 0165025410375921$.

Carlo, G., Mestre, V., Samper, P., Tur, A. M., \& Armenta, B. (2010b). Feelings or cognitions? Moral cognitions and emotions as longitudinal predictors of prosocial and aggressive behaviors. Personality and Individual Differences, 48(8), 865-962. doi:10.1016/j.paid.2010.02.010.

Cerezo, F. (2006). Violencia y victimización entre escolares. El bullying: estrategias de identificación y elementos para la intervención a través del Test Bull-S. Revista Electrónica de Investigación Psicoeducativa, 4(2), 333-352. rés se centra fundamentalmente en cómo perciben los adolescentes la relación con su padre y con su madre y cómo influye dicha relación en la victimización o el apego. En tercer lugar, se ha evaluado la percepción de victimización y de apego del adolescente, en próximos estudios se puede contrastar dicho autoinforme con la información de aceptación o rechazo por parte de los compañeros.

Consideramos que los resultados obtenidos sugieren líneas de intervención para la mejora de la convivencia escolar, la potenciación de un clima escolar positivo y el fomento del apoyo entre los compañeros.

Agradecimientos.- Este estudio ha sido posible gracias a la financiación del Proyecto I+D para grupos de investigación de excelencia (referencia PROMETEO 2011/009) Generalitat Valenciana; y del Proyecto I+D subvencionado por el Ministerio de Ciencia e Innovación (referencia PSI2011-27158).
Recuperado http://www.investigacionpsicopedagogica.org/revista/articulos/9/espannol/Art_9_115.pdf.

Cerezo, F. (2009). Bullying: análisis de la situación en las aulas españolas. International Journal of Psychology and Psychological Therapy, 9(3), 367-378. Recuperado en http://www.ijpsy.com/volumen9/num3/244/bullyinganalisis-de-la-situacion-en-las-ES.pdf.

Davis, M. H. (1980). A multidimensional approach to individual differences in empathy. JSAS Catalog of Selected Documents in Psychology, 10, 85. Recuperado de www.uv.es/ friasnav/Davis_1980.pdf.

Davis, M. H. (1983). Measuring individual differences in empathy: Evidence for a multidimensional approach. Journal of Personality and Social Psychology, 44(1), 113-126. doi: 10.1037/0022-3514.44.1.113.

Del Barrio, M. V., Moreno, C. y López, R. (2001). Evaluación de la agresión e inestabilidad emocional en niños españoles y su relación con la depresión. Clínica y Salud, 13, 33-50. Recuperado de: http:// www.redalyc.org/articulo.oa?id=180618320002.

Delgado, I., Oliva, A. y Sánchez-Queija, I. (2011). Apego a los iguales durante la adolescencia y la adultez emergente. Anales de Psicología, 27(1), 155-163. Recuperado de http:// revistas.um.es/analesps.

Eisenberg, N., Carlo, G., Murphy, B., \& Van Court, P. (1995). Prosocial Development in Late Adolescence: A Longitudinal Study. Child Development, 66(4), 1179-1197. doi: 10.2307/1131806.

Escobar, M., Fernández-Baena, F. J., Miranda, J., Trianes, M. V., \& Cowie, H. (2011). Low peer acceptance and emotional/behavioural maladjustment in schoolchildren: Effects of daily stress, coping and sex. Anales de Psicologia, 27(2), 412-417. Recuperado de http://revistas.um.es/analesps/article/view/123041/115911.

Estévez, E., Martínez, B. y Jiménez, T. I. (2009). Las relaciones sociales en la escuela: el problema del rechazo escolar. Psicología Educativa, 15(1), 512. Recuperado de http://www.uv.es/lisis/belen/psicolog-educt.pdf.

Frydenberg, E., \& Lewis, R. (1991). Adolescent coping: the different ways in which boys and girls cope. Journal of Adolescence, 14, 119-133. doi: 10.1016/0140-1971(91)90025-M.

Garaigordobil, M. (2010). El maltrato entre iguales: definición, prevalencia, consecuencias, identificación e intervención. Ponencia invitada en las Jornadas Formativas dirigidas a profesionales de la Psicología y la Educación que trabajan con alumnos/as con discapacidad intelectual. FEV AS. Federación V asca de Asociaciones a favor de las Personas con Discapacidad Intelectual.

Garaigordobil, M. y Oñaderra, J. A. (2010a). La violencia entre iguales: revisión teórica y estrategias de intervención. Madrid: Pirámide.

Garaigordobil, M. y Oñaderra, J. A. (2010b). Inteligencia emocional en las víctimas de acoso escolar y en los agresores. European Journal of Education and Psychology, 3(2), 243-256. Recuperado de www.ejep.es/index.php/journal/article/download/63/94. 
Garaigordobil, M. y Oñaderra, J. A. (2009). Acoso y violencia escolar en la comunidad autónoma del País Vasco. Psicothema, 21(1), 83-89. Recuperado de http://www.psicothema.com/pdf/3599.pdf.

Gini, G., Albiero, P., Benelli, B., \&Altoe, G. (2007). Does empathy predict adolescents' bullying and defending behavior? Aggressive Behavior, 33, 1 10. DOI: $10.1002 / \mathrm{ab} .20204$

Graham, S. (2006). Peer victimization in school. Exploring the ethnic context. Current Directions in Psychological Science, 15(6), 317-321. doi: 10.1111/j.1467-.

Herrero, J., Estévez, E., \& Musitu, G. (2006). The relationships of adolescent school-related deviant behavior and victimization with psychological distress: Testing a general model of the meditational role of parents and teachers across groups of gender and age. Journal of Adolescence, 29, 671-690. doi: 10.1016/j.adolescence.2005.08.015.

Hu, L., \& Bentler, P. M. (1999). Cutoff criteria for fit indexes in covariance structure analysis: Conventional criteria versus new alternatives. Structural Equation Modeling, 6, 1-55 doi: DOI:10.1080/10705519909540118.

Ison-Zintilini, M. S. y Morelato, G. S. (2008). Habilidades socio-cognitivas en niños con conductas disruptivas y víctimas de maltrato. Universitas Psychologica, 7(2), 357-367. Recuperado de http://www.scielo.org.co/pdf/rups/v7n2/v7n2a05.pdf.

Kokkinos, C. M., \& Kipritsi, E. (2012). The relationship between bullying, victimization, trait emotional intelligence, self-efficacy and empathy among preadolescents. Social Psychological Education, 15, $42-58$ doi: 10.1007/s11218-011-9168-9.

Malti, T., Perren, S., \& Buchmann, M. (2010). Children's peer victimization, empathy, and emotional symptoms. Child Psychiatry of buman Development, 41, 98-113 DOI 10.1007/s10578-009-0155-8.

Martínez-Ferrer, B., Povedano-Díaz, A., Amador-Muñoz, L. V. y MorenoRuiz, D. (2012). Clima escolar, satisfacción con la vida y victimización en la escuela. Un análisis del efecto moderador del género. Anales de Psicología, 28(3), 875-882. doi: 10.6018/analesps.28.3.156121.

Mavroveli, S., Petrides, K. V., Sangareau, Y., \& Furnham, A. (2009). Exploring the relationships between trait emotional intelligence and objective socio-emotional outcomes in childhood. British Journal of Educational Psychology, 79, 259-272 doi: 10.1348/000709908X368848.

Mestre, V., Frías, M. D. y Samper, P. (2004). La medida de la empatía: análisis del Interpersonal Reactivity Index. Psicothema, 16(2), 255-260. Recuperado http://www.unioviedo.es/reunido/index.php/PST/article/view/8243 $/ 8107$.

Mestre, V., Samper, P. y Frías, M. D. (2002). Procesos cognitivos y emocionales predictores de la conducta prosocial y agresiva: la empatía como factor modulador. Psicothema, 14, 227-232. Recuperado de http://www.psicothema.com/pdf/713.pdf.

Mestre, V., Samper, P., Nácher, M. J, Tur, A., \& Cortés, M. T (2006). Psychological processes and family variables as prosocial behavior predictors in a sample of Spanish adolescents. Psychological Report, 98, 30-36. doi: $10.2466 / \mathrm{pr} 0.98 .1 .30-36$.
Mestre, V., Samper, P., Tur-Porcar, A., Richaud, C. y Mesurado, B. (2012). Emociones, Estilos de Afrontamiento y Agresividad en la adolescencia. Universitas Psychologica, 11(4), 1263-1275. Recuperado de http://revistas.javeriana.edu.co/index.php/revPsycho/article/view/12 $63 / 3356$

Mestre, V., Tur, A., Samper, P., Nácher, M. J. y Cortés, M. T. (2007). Estilos de crianza en la adolescencia y su relación con el comportamiento prosocial. Revista Latinoamericana de Psicología, 39(2), 211-225. Recuperado de http:/ / www.scielo.org.co/scielo.php?pid=S0120 05342007000200001\&script $=$ sci_arttext.

Oliva, A., Parra, A. y Arranz, E. (2008). Estilos relacionales parentales y ajuste del adolescente. Infancia y Aprendizaje, 31(1), 93-106. DOI: http://dx.doi.org/10.1174/021037008783487093.

Olweus, D. (1993). Victimization by peers: antecedents and long-term outcomes. En K. H. Rubin y J. B. Asendorf (Eds) Social withdrawal, inbibition, and shyness in childhood (pp.315-341). Hillsdale, N.J.: Lawrence Erlbaum.

Ortiz, M. J., Apodaca, P., Etxebarría, I., Fuentes, M. J. y López, F. (2008) Predictores familiares de la internalización moral en la infancia. Psicot bema, 20(4), 712-717. Recuperado de $<$ http:/ / www.redalyc.org/articulo.oa?id $=72720431>$

Pereña, J. y Seisdedos, N. (1997). ACS. Escalas de afrontamiento para Adolescentes. Madrid: TEA.

Petrides, K. V., Frederickson, N., \& Furnham, A. (2004). The role of trait emotional intelligence in academic performance and deviant behavior at school. Personality and Individual Differences, 36, 277-293. DOI http://dx.doi.org/10.1016/S0191-8869(03)00084-9.

Piñero-Ruiz, E. López-Espín, J. J., Cerezo, F. y Torres-Cantero, A. M. (2012). Tamaño de la fratría y victimización escolar. Anales de Psicología, $28(3)$ $842-847$. Recuperado $<$ http:/ / www.redalyc.org/articulo.oa?id=16723774022>

Samper, P., Cortés, M. T., Mestre, M. V., Nácher, M. J. y Tur, A. M. (2006). Adaptación del Child's Report of Parent Behavior Inventory a población española. Psicothema, 18(2), 263-271. Recuperado de: $<$ http:/ /www.redalyc.org/articulo.oa?id $=72718216>$ I

Sánchez, V., Ortega, R. y Menesini, E. (2012). La competencia emocional de agresores y víctimas de bullying. Anales de psicología, 28(1), 71-82. Recuperado de revistas.um.es/analesps/article/download/140542/126632.

Schaefer, E. S. (1965). Children's Reports of Parental Behavior: an inventory. Child Development, 36, 413-424. doi: 10.2307/1126465.

Tur-Porcar, A., Mestre, V, Samper, P. y Malonda, E. (2012). Crianza y agresividad de los menores: ¿es diferente la influencia del padre y de la madre? Psicothema, 24(2), 284-288 Recuperado de: $<$ http:/ / www.redalyc.org/articulo.oa?id $=72723578017>$.

Wahl, K., \& Metzner, C. (2012). Parental Influences on the Prevalence and Development of Child Aggressiveness. Journal of Child and Family Studies, 21, 344-355. DOI 10.1007/s10826-011-9484-x.

(Articulo recibido: 24-04-2013; revisado: 06-11-2013; aceptado: 24-05-2014) 